\title{
Phrenic nerve palsy after internal jugular venous catheter placement
}

\author{
Eun Jin Ahn, Chong Wha Baek, Hwa Yong Shin, Hyun Kang, and Yong Hun Jung \\ Department of Anesthesiology and Pain Medicine, College of Medicine, Chung-Ang University, Seoul, Korea
}

Central venous catheters are usually inserted to measure the central venous pressure or use as an administration route for fluid or medication. There are reports that complications, such as arterial puncture, pneumothorax, occur in approximately $15 \%$ of cases when inserting it $[1,2]$. Phrenic nerve palsy is a very rare complication attributed to central venous catheter insertion. It can be caused when inserting the catheter into the subclavian vein or internal jugular vein, as well as when venal puncture is attempted several times with a thick needle, and can also occur when the needle is inserted too deeply, directly injuring the nerves. We recently had a case of phrenic nerve palsy, which was discovered late and its cause suspected to be central venous catheter insertion.

The patient, a 75 year old female, underwent left nephroureterectomy for papillary urothelial carcinoma. At the time, there were no abnormalities in the preoperative physical examinations and laboratory tests including a chest x-ray. A central venous catheter was inserted through land mark guidance, by means of the anterior approach, into the right internal jugular vein [1]. There were no difficulties or specific events during the procedure. The operation proceeded without any complications, yet the chest $\mathrm{x}$-ray taken on the same day after the surgery, indicated pulmonary congestion and atelectasis on the right lower lobe. Two days after the surgery, the central venous catheter was removed. The ABGA, performed on the third day post surgery showed $\mathrm{pH} 7.42, \mathrm{PaCO}_{2} 40.7$ $\mathrm{mmHg}$, and $\mathrm{PaO}_{2} 64.1 \mathrm{mmHg}$ in room air and the chest x-ray showed no differences when compared to the one before. Ten days after the surgery, the patient was discharged with no specific symptoms and was advised to take deep breaths and cough.

Six months later, the patient visited the outpatient clinic with dyspnea, which began occurring a month prior without any other symptoms, such as coughing or sputum. Atelectasis in the right lower lobe was observed in the chest x-ray taken, which was similar to the chest $\mathrm{x}$-ray from six months before. In the dynamic chest $\mathrm{CT}$, the right diaphragm had risen and segmental atelectasis could be observed in the right middle and lower lobes. Both an electroneurography and electromyography were performed on the right upper and lower limbs under the suspicion of phrenic nerve palsy. The results showed an abnormal electrophysiological finding that suggested right cervical radiculopathy (C 4, 5, 7) and right phrenic neuropathy. The right median nerve, ulnar nerve, lower limb nerve, and left phrenic nerve, were all normal. In the Jolly test performed, in order to rule out abnormalities in the neuromuscular junction, no abnormal electrophysiological findings were observed. The patient did not complain of other symptoms in the upper or lower limbs, other than dyspnea. Also, with the exception of the history of central venous catheter insertion six months prior, there were no other causes for the phrenic nerve palsy, such as tumor or infection. Hence, the central venous catheter insertion was suspected as the most probable cause for the phrenic nerve palsy.

The patient complained of dyspnea continuously, so a diaphragm plication was performed. The pulmonary function test, performed nine days after the surgery, showed FVC 1,510 (67\%) $\mathrm{ml}$ and $\mathrm{FEV}_{1} 1,030(68 \%) \mathrm{ml}$, which had improved from

Corresponding author: Chong Wha Baek, M.D., Department of Anesthesiology and Pain Medicine, College of Medicine, Chung-Ang University, 224-1, Heukseok-1dong, Dongjak-gu, Seoul 156-756, Korea. Tel: 82-2-6299-2583, Fax: 82-2-6299-2585, E-mail: nbjhwa@naver.com

(c) This is an open-access article distributed under the terms of the Creative Commons Attribution Non-Commercial License (http:// creativecommons.org/licenses/by-nc/3.0/), which permits unrestricted non-commercial use, distribution, and reproduction in any medium, provided the original work is properly cited. 
the pulmonary function test performed before the surgery with FVC 1,350 (60\%) $\mathrm{ml}$ and $\mathrm{FEV}_{1} 950$ (63\%) ml. The dyspnea improved twelve days after the surgery, so the patient was discharged, and currently, six months after the final surgery, the patient has had no other problems.

Other than direct damage inflicted by the needle, phrenic nerve palsy can also occur from the nerve being pressed by blood clots, or, from a successfully inserted central venous catheter pressing against the phrenic nerve, which moves along the superior vena cava [3]. Phrenic nerve palsy can temporarily appear as a result of local anesthetics infiltrating the subcutaneous in an effort to reduce the pain of central venous catheter insertion in patients who are awake. Nerve damage can be accelerated in patients with diabetic polyneuropathy, since inflammation develops on the vessel that supplies blood flow to the phrenic nerve [3].

To reduce the rate of complication occurrences, such as phrenic nerve palsy, which occurs when inserting a central venous catheter, the indications for the central venous catheter insertion should be carefully considered first, and the practitioner should be well acquainted with the anatomic structures surrounding the central vein. Complications are mostly caused by inexperienced one in a difficult case of central venous catheter insertion. Especially, when an attempt on one side fails and is reattempted on the other side. It can lead to bilateral phrenic nerve injury requiring complicated treatments. When an ultrasound guide is used, although it is difficult to find small nerves like the phrenic nerve, nerve relationships can be known through the surrounding structures, such as muscles and blood vessels. By knowing these structural relationships, it is helpful in apprehending the location of the phrenic nerve. In summary, it is advised that ultrasound guidance is used accompanied by the use of thin needles with careful concern of an expert. After inserting the catheter, as in the case of our patient, a chest $\mathrm{x}$-ray showing an ascended right diaphragm can be mistaken for segmental atelectasis. As a result, it is always important to keep in mind the possibility of complications, and to also be well aware that careful observations and aggressive treatment should be administered according to the changes in the patient's state and chest $\mathrm{x}$-rays.

\section{References}

1. Feller-Kopman D. Ultrasound-guided internal jugular access: a proposed standardized approach and implications for training and practice. Chest 2007; 132: 302-9.

2. Boon JM, van Schoor AN, Abrahams PH, Meiring JH, Welch T. Central venous catheterization --an anatomical review of a clinical skill. Part 2. Internal jugular vein via supraclavicular approach. Clin Anat 2008; 21: 15-22.

3. Paraskevas GK, Raikos A, Chouliaras K, Papaziogas B. Variable anatomical relationship of phrenic nerve and subclavian vein: clinical implication for subclavian vein catheterization. Br J Anaesth 2011; 106: 348-51. 


\section{Notice}

1. On-line manuscript review process

1) All proposed manuscripts for publication in the Korean Journal of Anesthesiology will be processed exclusively online.

Please use the "Questions and Answers" section of our website (http://submit.ekja.org/) for any concerns you may have vis-á-vis problems, questions, or suggestions for improvement. You may also direct them to the attention of the electronic manuscripts processing manager (editorial manager) (MEDrang, +82-2-6711-4711).

2) Processing publication-related inquiries for newly submitted manuscripts through the main number of the KJA would cause undue burden for the KJA in its general duties.

As such, these inquiries should be made entirely online in the "Questions \& Answers" section of the website. Requests and orders - including the expected date of publication and the status of manuscript under review - should be made online, and responses shall be provided without delay upon confirmation.

\section{Information and regulation for submitting manu-} scripts

1) The corresponding author will be notified by e-mail whenever there is any change in the status of a submitted manuscript. Authors could also obtain this information directly through the website. The KJA will respond to all review inquiries. However, the expected Vol. No. cannot be provided, if it has not yet been confirmed; as such, we would not be able to entertain inquiries on this matter.

2) Doctoral and Master's dissertations should abide by the manuscript submission guidelines of the KJA and should accompany a scanned file of the thesis title and the signature(s) of the examiner(s). This information should be entered in the "Paper Information" section during the online submission process.

3) The corresponding author should be a faculty. The corresponding author will be notified by e-mail whenever there is any change in the status of a submitted manuscript, and any resubmission can only be made by the corresponding author.

4) A manuscript needs to be resubmitted if there is a change in the makeup of the manuscript authorship; thus, special care should be taken when registering authors.

5) Manuscript review is terminated after two reviews, but a member of the review committee may request for a 3 rd review.

3. How to publish color images on www.ekja.org Color images in the printed journal require an additional expense. 